\title{
Energy vs. density on paths toward exact density functionals
}

\section{Kepp, Kasper Planeta}

\section{Published in:}

Physical Chemistry Chemical Physics

Link to article, DOI:

10.1039/C7CP07730K

Publication date:

2018

Document Version

Peer reviewed version

Link back to DTU Orbit

Citation (APA):

Kepp, K. P. (2018). Energy vs. density on paths toward exact density functionals. Physical Chemistry Chemical Physics, 20(11), 7538-7548. https://doi.org/10.1039/C7CP07730K

\section{General rights}

Copyright and moral rights for the publications made accessible in the public portal are retained by the authors and/or other copyright owners and it is a condition of accessing publications that users recognise and abide by the legal requirements associated with these rights.

- Users may download and print one copy of any publication from the public portal for the purpose of private study or research.

- You may not further distribute the material or use it for any profit-making activity or commercial gain

- You may freely distribute the URL identifying the publication in the public portal

If you believe that this document breaches copyright please contact us providing details, and we will remove access to the work immediately and investigate your claim. 

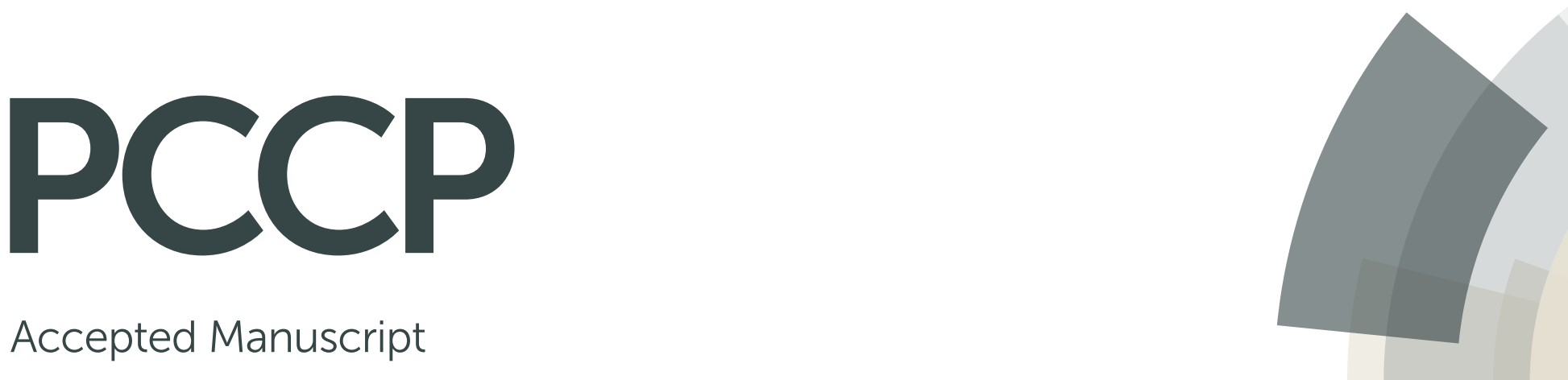

Accepted Manuscript

This article can be cited before page numbers have been issued, to do this please use: K. P. Kepp, Phys. Chem. Chem. Phys., 2018, DOI: 10.1039/C7CP07730K.

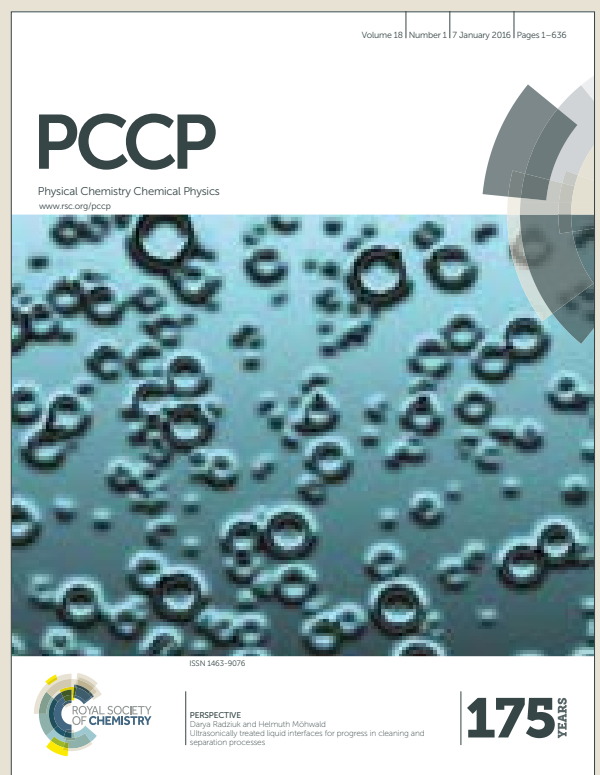

This is an Accepted Manuscript, which has been through the Royal Society of Chemistry peer review process and has been accepted for publication.

Accepted Manuscripts are published online shortly after acceptance, before technical editing, formatting and proof reading. Using this free service, authors can make their results available to the community, in citable form, before we publish the edited article. We will replace this Accepted Manuscript with the edited and formatted Advance Article as soon as it is available.

You can find more information about Accepted Manuscripts in the author guidelines.

Please note that technical editing may introduce minor changes to the text and/or graphics, which may alter content. The journal's standard Terms \& Conditions and the ethical guidelines, outlined in our author and reviewer resource centre, still apply. In no event shall the Royal Society of Chemistry be held responsible for any errors or omissions in this Accepted Manuscript or any consequences arising from the use of any information it contains. 


\title{
Energy vs. density on paths toward more exact density functionals
}

\author{
Kasper P. Kepp* \\ Technical University of Denmark, DTU Chemistry, Building 206, 2800 Kgs. Lyngby, DK - \\ Denmark. *Phone: +045 452524 09. E-mail: kpj@kemi.dtu.dk
}

\begin{abstract}
Recently, the progression toward more exact density functional theory has been questioned, implying a need for more formal ways to systematically measure progress, i.e. a "path". Here I use the Hohenberg-Kohn theorems and the definition of normality by Burke et al. to define a path toward exactness and "straying" from the "path" by separating errors in $\rho$ and $E[\rho]$. A consistent path toward exactness involves minimizing both errors. Second, a suitably diverse test set of trial densities $\rho^{\prime}$ can be used to estimate the significance of errors in $\rho$ without knowing the exact densities which are often inaccessible. To illustrate this, the systems previously studied by Medvedev et al., the first ionization energies of atoms with $Z=1$ to 10 , the ionization energy of water, and the bond dissociation energies of five diatomic molecules were investigated using $\operatorname{CCSD}(\mathrm{T}) /$ aug-cc-pV5Z as benchmark at chemical accuracy. Four functionals of distinct designs was used: B3LYP, PBE, M06, and S-VWN. For atomic cations regardless of charge and compactness up to $Z=10$, the energy effects of the different $\rho$ are $<4 \mathrm{~kJ} / \mathrm{mol}$ (chemical accuracy) defined here as "normal", even though these four functionals ranked very differently in the previous test. Thus, the "off-path" behavior for such cations is energy-wise insignificant. An interesting oscillating behavior in the density sensitivity is observed vs. $Z$, explained by orbital occupation effects. Finally, it is shown that even large "normal" problems such as the Co-C bond energy of cobalamins can use simpler (e.g. PBE) trial densities to drastically speed up
\end{abstract}


computation by loss of a few $\mathrm{kJ} / \mathrm{mol}$ in accuracy. The proposed method of using a test set of trial densities to estimate the sensitivity and significance of density errors of functionals may be useful for testing and designing new balanced functionals with more systematic improvement of densities and energies.

Keywords: density functional theory, Hohenberg-Kohn Theorem, accuracy, exact functional, ionization energy, trial density 


\section{Introduction}

A major challenge in current theoretical chemistry is the development of new, more generally applicable and accurate functionals, because all functionals lack general high accuracy: Some are accurate for some electronic systems, others are for other systems, and the search for "universal" accuracy is thus ongoing ${ }^{1-6}$. An exact functional has a negligible error (i.e. smaller than the uncertainty in standard approximations beyond the functional itself) in both the density $\rho$ and its derived properties, notably the associated energy $E[\rho]$, for a given electronic system (numerically exact); in addition it should fulfil all fundamental exact physical conditions (fundamentally exact). The universal functional is the functional that is exact for any system and for any physical observable ${ }^{7} . E[\rho]$ itself is not observable, except in principle by reverse mapping from an experimental $\rho$ obtained by e.g. X-ray diffraction using the universal functional, via the one-to-one correspondence ${ }^{4}$. Thus, accuracy of a functional is typically assessed by the errors in derived energies of the type $\Delta E=E_{2}\left[\rho_{2}\right]-E_{1}\left[\rho_{1}\right]$ where $\Delta E$ represents a chemical conversion whose energy change is observable, e.g. the ionization potential, with a target accuracy of $\sim 4$ $\mathrm{kJ} / \mathrm{mol}$ (chemical accuracy).

In a recent report ${ }^{2}$ it was claimed that modern density functionals are becoming less exact. Specifically, the paper indicated that some relatively new, highly parameterized functionals produce less accurate $\rho$ for charged $1 s^{2}$ and $1 s^{2} 2 s^{2}$ atomic ions, although these functionals often perform well for derived energies $\Delta E[\rho]$. From this observation it was concluded that modern DFT is straying from the path toward exactness ${ }^{2}$. The debate goes to the very center of the theory, specifically the Hohenberg-Kohn correspondence between the electron density $\rho$ and its derived properties ${ }^{4}$. Functionals can be accurate for one property but inaccurate for another property for the same system ${ }^{5,8,9}$. To speak of a path toward exactness, errors must be assessed not only in $\rho$ but also $E[\rho]$ for the same systems, because exactness applies to a specific 
system $^{10}$. In fact, the errors of $\rho$ and $\Delta E[\rho]$ are commonly not related even for the same systems except in very distinct simple cases such as the closed-shell $2 \mathrm{~s}^{2}$ double ionization energies of $1 s^{2} 2 s^{2}$ systems ${ }^{10}$; thus, many functionals produce decent $\rho$ (in terms of their ability to serve as trial densities for more exact functionals) but inaccurate $\Delta E[\rho]^{11}$. These two properties are particularly central to DFT because they feature in the Hohenberg-Kohn theorems as having a one-to-one correspondence ${ }^{4}$, where $\rho$ obeys the variational principle and is $\mathrm{N}$ - and vrepresentable $\mathrm{e}^{3,12}$.

A relevant question is whether there is a monotonic path toward exactness as $\rho \rightarrow \rho_{\text {exact }}$ and $E[\rho] \rightarrow E_{\text {exact }}\left[\rho_{\text {exact }}\right]$. To visualize this one can plot errors in $\rho$ and $E[\rho]$ in a diagram such as Figure 1A, where a path is given when and only when $\rho \rightarrow \rho_{\text {exact }}$ and $E[\rho] \rightarrow E_{\text {exact }}\left[\rho_{\text {exact }}\right]$ for the same electronic system. In the case of no improvement in $\rho$ but only in $E[\rho]$, we move horizontally to the right in Figure 1A, and in the reverse case we move straight upwards. Those paths resemble conversions in a thermodynamic cycle, because $E[\rho]$ is a state function. Thus, as a definition, a path toward exactness is a variation in $\rho$ and $E[\rho]$ that improves $\rho$ and/or $E[\rho]$ without deteriorating the other. Such a definition seems required to assess whether there is any "straying" from a path toward accuracy. In the above numerical definition of exactness, this path is well-defined by the errors of $\rho$ and $\Delta E[\rho]$, which is an observable functional of $\rho$ (which $E[\rho]$ is not).

Straying from the path is then any change in a functional that increases error in $\rho$ or $\Delta E[\rho]$. Accordingly, the previously discussed straying ${ }^{2}$ can be seen to represent the dashed arrows on the diagonal from the top left toward the bottom right of Figure 1A. The direct path toward exactness is defined as the path that reduces errors in $\rho$ or $E[\rho]$ by the same proportion, represented by the diagonal lines moving from bottom right towards top left. It is an important special type of transformation of $\rho \rightarrow \rho_{\text {exact }}$ and $E[\rho] \rightarrow E_{\text {exact }}\left[\rho_{\text {exact }}\right]$ where both transform by the 
same scale factor. Functionals that follow this path can be set to be "balanced". One may improve functionals by adding more mathematical terms of the gradient expansions, or by reducing errors vs. benchmark data sets, or by obeying more exact bounds (functionals marked " and ' in Figure 1A), or ideally several of these features in combinations, with some improving $\rho$ more than the $E[\rho]$ or vice versa. These paths will deviate from the direct path but are not "straying" but rather converging toward exactness.

It is now useful to consider all real functionals as trial functionals of the exact functional. For the energy of any trial density $\rho^{\prime}$ studied by another functional $E\left[\rho^{\prime}\right]^{4}$,

$$
E\left[\rho^{\prime}\right] \geq E_{0}
$$

where $E_{0}$ is the lowest possible energy (or "ground state" energy) $E[\rho]$ obtained from that functional with its variationally optimized $\rho$. Thus, any other $\rho^{\prime}$ will produce a larger $E\left[\rho^{\prime}\right]$. Because of equation (1), the native combination, e.g. $E^{\prime}\left[\rho^{\prime}\right]$, is always lower in energy than any of the states vertically above or below it, e.g. $E^{\prime}\left[\rho^{\prime \prime}\right]$ and even $E^{\prime}\left[\rho_{\text {exact }}\right]$. By inspection of Figure 1A, the top right horizontal process corresponds to minus the functional-derived error, $-\Delta E_{\mathrm{F}}{ }^{\prime}=$ $E_{\text {exact }}\left(\rho_{\text {exact }}\right)-E^{\prime}\left(\rho_{\text {exact }}\right)$ for the functional $E^{\prime}\left[\rho^{\prime}\right]$ in the definition by Burke and co-workers ${ }^{11}$, whereas the left vertical process of the upper right cycle corresponds to minus the densityderived error, $-\Delta E_{\mathrm{D}}{ }^{\prime}=E^{\prime}\left(\rho_{\text {exact }}\right)-E^{\prime}\left(\rho^{\prime}\right) \geq 0$, where the last inequality follows from the variational Hohenberg-Kohn principle, with equal being the case only for $\rho^{\prime}=\rho_{\text {exact }}$. Burke and co-workers already discussed that a majority of systems behave normally (at least beyond Thomas-Fermi theory) in the sense that $\Delta E_{\mathrm{D}}{ }^{\prime}<<\Delta E_{\mathrm{F}}$. 


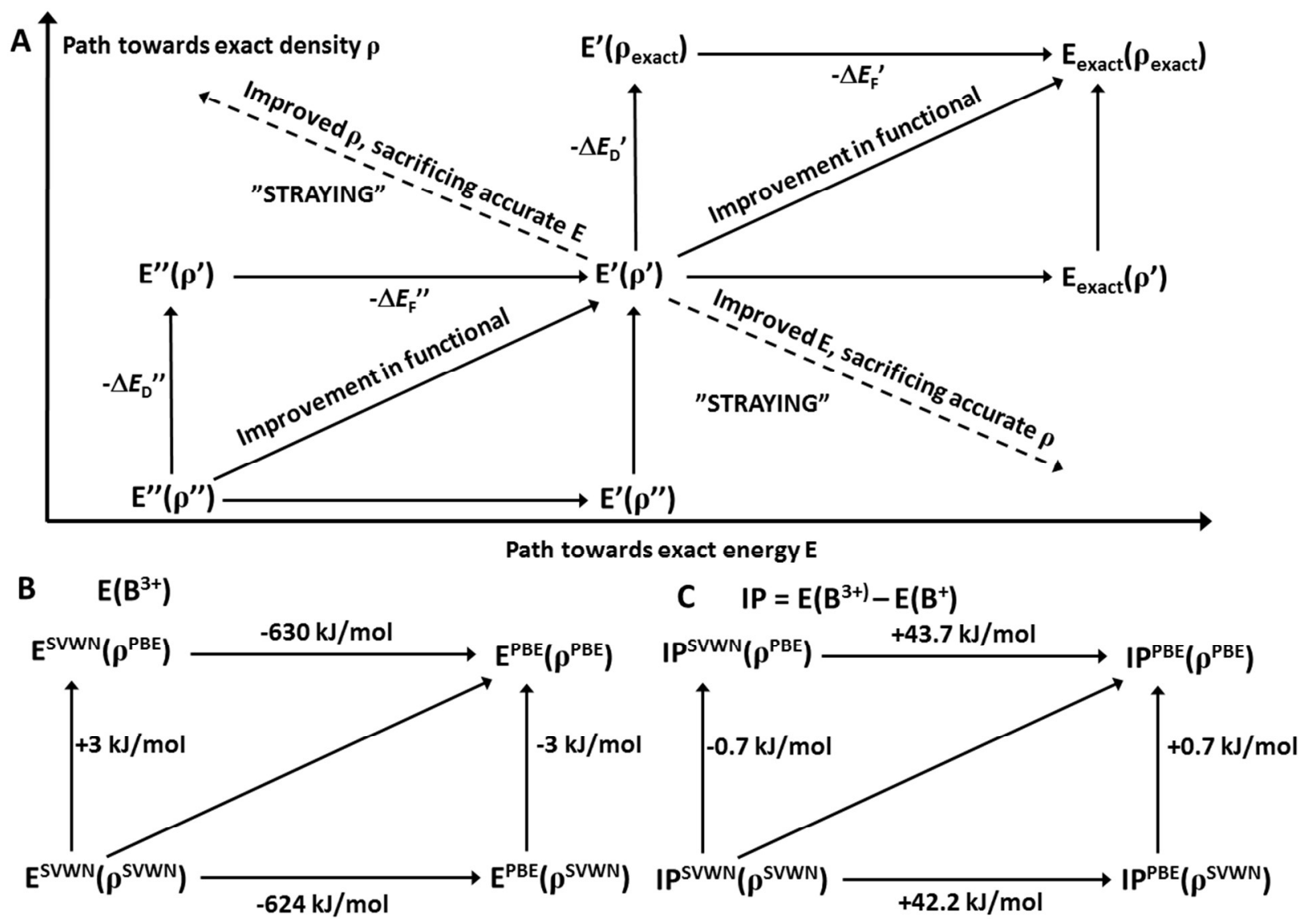

Figure 1. Simplified scheme of the paths toward more accurate density functionals. A) Definition of the path toward exactness for both energy and density; B) example with the absolute energy of $\mathrm{B}+$; C) example with the derived, experimentally observable energy $\mathrm{E}\left(\mathrm{B}^{3+}\right)$ $\mathrm{E}\left(\mathrm{B}^{+}\right)$

The present work uses the Hohenberg-Kohn trial density concept and the fact that $E[\rho]$ is a state function to estimate the chemical significance of errors in $\rho$ as an alternative to using normalized relative errors that may not show the energy impact directly ${ }^{2}$. The definitions of paths toward or away from exactness in Figure 1 may help in defining norms for calculation. To do so, we can evaluate (without using costly correlated wave function methods) the chemical significance of errors in $\rho$ that are intensively discussed ${ }^{2}$. More specifically, categorizing 
electronic systems and functionals as practically normal or abnormal based on the sensitivity of energies to variations in trial densities (i.e. $\mathrm{E}\left[\rho^{\prime}\right]-\mathrm{E}[\rho]$ ) rather than errors vs. the elusive exact density) turns out to be useful since exact densities are computationally inaccessible for most electronic systems. To demonstrate the protocol, it is first applied to the previously studied compact ions $^{2}$, and subsequently to the more chemically relevant first ionization potentials of atoms and the bond dissociation energies of molecules. It is shown that "practical normality" is a good proxy for "exact normality" and all the studied electronic systems are "practically normal", i.e. even large variations in densities produce insignificant energy variations, and thus the systems are not good norms for estimating progress on the path defined in Figure 1.

\section{Methods.}

All computations were performed using the Turbomole software, version $7.0^{13}$ and the resolution of identify approximation was used to speed up all calculations ${ }^{14,15}$. For the native combinations of methods (i.e. those using their own converged $\rho$ ), all densities and energies were converged to $10^{-7}$ a.u. The energies of all molecules, ions, and atoms were computed using the aug-cc-pV5Z basis $\operatorname{set}^{16}$ (numerical data in Table $\mathrm{S} 1$ ), except the atomic ions $\mathrm{B}^{+}, \mathrm{B}^{3+}, \mathrm{C}^{2+}, \mathrm{C}^{4+}, \mathrm{N}^{3+}, \mathrm{N}^{5+}, \mathrm{O}^{4+}$,

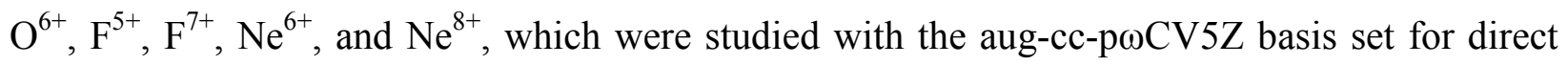
comparison to previous studies using this basis $\operatorname{set}^{2,10}$. To investigate the effect of basis set on $\rho$ variations and $\Delta E_{\mathrm{D}}$ ', the def-TZVP basis $\operatorname{set}^{17}$ was also used for some systems.

Ionization potentials (IP) were computed as:

$$
\mathrm{IP}(\mathrm{X})=E_{\mathrm{el}}\left(\mathrm{X}^{+}\right)-E_{\mathrm{el}}(\mathrm{X})
$$

where $E_{\text {el }}$ represents the electronic energy obtained with a method using a specified frozen density obtained by a converged previous computation either by the same method or another 
method. The double-ionization potentials (not to be confused with the second ionization potentials) studied for $\mathrm{B}^{+}, \mathrm{C}^{2+}, \mathrm{N}^{3+}, \mathrm{O}^{4+}, \mathrm{F}^{5+}$, and $\mathrm{Ne}^{6+}$ were computed as described previously ${ }^{10}$ :

$$
\mathrm{IP}\left(\mathrm{X}^{\mathrm{n}+}\right)=E_{\mathrm{el}}\left(\mathrm{X}^{(\mathrm{n}+2)+}\right)-E_{\mathrm{el}}\left(\mathrm{X}^{\mathrm{n}+}\right)
$$

The advantage of these experimentally available energies (they can be derived from ionization potentials of various order ${ }^{10}$ ) is that they involve strict comparison of the quality of $E[\rho]$ of the same studied closed-shell $1 \mathrm{~s}^{2}$ and $1 \mathrm{~s}^{2} 2 \mathrm{~s}^{2}$ configurations without a need to invoke additional open-shell configurations, as would be the case for first ionization potentials.

The bond dissociation energies $(B D E)$ were computed as:

$$
B D E(X Y)=E_{\mathrm{el}}(X)+E_{\mathrm{el}}(Y)-E_{\mathrm{el}}(X Y)-Z P E(X Y)
$$

where $E_{\mathrm{el}}(X)$ is the electronic energy of species $\mathrm{X}$ computed and $Z P E(\mathrm{XY})$ is the vibrational zero-point energy of $\mathrm{XY}$. In some cases, only the energies without $Z P E$ were compared as $Z P E$ does not affect the study of $\rho$ because $Z P E$ is a constant of the geometry optimized with BP86/def2-TZVPP and generally changes only $1-2 \mathrm{~kJ} / \mathrm{mol}^{\text {with method }}{ }^{18,19}$. The BDEs and IPs represent the most important simple proxies of "real" chemistry, possibly together with electron affinities. However, the latter suffer from being commonly affected by self-interaction errors and basis set artifacts of the diffuse anion states. The purpose of this work, in addition to using the definitions in Figure 1, was to specifically show that previously suggested norms for studying density variations display insignificant energy effects because they are practically normal. Burke et al. has already argued that electron affinities and other systems likely to suffer from self interaction error may be abnormal, and such systems were avoided for scope and clarity ${ }^{20,21}$.

The geometry used to compute the Co-C BDE was the complete, large cobalamin model including side chains that was previously published ${ }^{22}$. For def2-TZVPP this involves 4538 basis functions for the calculation of the energies of the complex and 338 closed-shell doubly occupied 
MOs (3710 basis functions for the cofactor radical where the 5'-deoxyadenosyl group has dissociated). The BDEs were in these cases computed using the conductor-like screening model $(\mathrm{Cosmo})^{23}$ as implemented in Turbomole ${ }^{24}$ with $\varepsilon=80$ and the dispersion D3 correction term by Grimme $^{25}$ except for SVWN, to produce a more realistic estimate of the true Co-C BDE, which depends on solvation and dispersion effects ${ }^{22}$ (which the diatomic data do not since they are known experimentally in vacuum and dispersion effects are $<1 \mathrm{~kJ} / \mathrm{mol}$ on the BDEs of these simple diatomic molecules ${ }^{26}$ ).

To identify "practically normal" systems and thereby estimate the significance of errors and deviations from the path in Figure 1, the recipe is to choose a small but diverse trial set of e.g. four functionals, ideally from four different rungs in Jacob's ladder ${ }^{27}$. It is assumed that the standard deviations in $E\left[\rho^{\prime}\right]$ within this set are then good estimates of the energy effects of varying $\rho$. If local functionals such as SVWN are included, this is probably correct (assuming that Jacob's ladder implies increased exactness). To illustrate the protocol, four diverse density functionals were chosen for the main trial set: B3LYP, PBE, SVWN, and M06. B3LYP ${ }^{28-30}$ represents a lightly parameterized hybrid GGA functional with 25\% HF exchange, and M06 represents a heavily parameterized meta hybrid with $27 \% \mathrm{HF}$ exchange ${ }^{31}$, PBE is a GGA nonhybrid $^{32}$, and SVWN is composed of the Slater exchange functional and the local VWN correlation functional $^{33}$ and thus represents the Local Spin Density Approximation (LSDA). These functionals ranked very differently in terms of the accuracy for $\rho$ of small atomic ions, with the largest maximum normalized error in $\rho$ seen for SVWN (3.725), with the other three spreading conveniently at 2.495 (PBE), 2.123 (B3LYP), and 1.838 (M06) $)^{2}$. Accordingly, both numerically and algorithmically they represent four very distinct functionals as desired to estimate fairly the sensitivity of E towards $\rho$-variations. Subsequently, the results were validated by using an even more diverse (in terms of $\rho$-variations) trial test set consisting of M06-2X 
(1.463), PBE (2.495), SVWN (3.725), and PBE0 (1.675), with maximum errors in $\rho$ reported in parenthesis from previous work ${ }^{2}$.

In order to study numerically the effect of $\rho$ vs. $E[\rho]$, the trial density concept was used in the following way: In addition to all "native" computations $E[\rho]$, computations were also done for all combinations of $E\left[\rho^{\prime}\right]$ and $E^{\prime}[\rho]$ for the trial test set, where $E$ and $E^{\prime}$ signifies energies obtained from two different functionals using fixed densities obtained by previous convergence with the other functional. For a trial set of $N$ functionals, this implies $N^{2}$ energy calculations for each electronic system. Fixed densities were obtained by allowing only a single energy evaluation of the new functional on the converged density and having infinite thresholds for convergence of the density (\$denconv in Turbomole). The densities along an axis (x) of the coordinate system of the atom or atomic ion were in all cases printed to text files and it was confirmed that densities were fixed and identical after calculation when used as trial densities.

\section{Results and Discussion.}

Using Trial Densities To Estimate p-Derived Errors. The formally defined error ${ }^{11}$ in a functional due to its density, $\Delta E_{\mathrm{D}}$ ' (upper vertical process in Figure 1A) is not known unless $\rho_{\text {exact }}$ and $E_{\text {exact }}\left[\rho_{\text {exact }}\right]$ is known, which is only approximately the case for very small electronic systems that can be computed by correlated methods such as $\operatorname{CCSD}(\mathrm{T})$ with extensive basis sets. Even this method may fail for more complex and relevant electronic systems (e.g. d-block elements, where CCSDT or multi-reference methods may be required). In fact, even for the very small dense cations studied previously, the CCSD method may not be exact non-relativistic, because of assumptions such as the use of Gaussian basis functions, which have the wrong structure exactly at the nuclei where most of the density error is localized. A simple comparison 


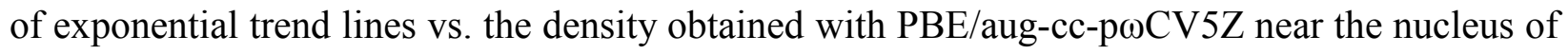
$\mathrm{B}^{+}$shows effects of 0.5 a.u or almost $1 \%$ of the total density at the nucleus (Figure S1). For the gradient and Laplacian the use of Gaussian basis functions to evaluate density errors for charged ions may be critical.

Because of this obstacle, we cannot systematically improve functionals along both axes of Figure 1A using $\Delta E_{\mathrm{D}}$ ' directly. Instead, I define a modified version of the normality proposed by Burke, which was based on the full error vs. the exact functional. To do so I use the same definition applied to the left thermodynamic cycle in Figure 1A. In any study of a non-exact functional $E^{\prime \prime}\left[\rho^{\prime \prime}\right]$ (which will always be the case), we can compute exactly the corresponding value $E^{\prime \prime}\left[\rho^{\prime}\right]-E^{\prime \prime}\left[\rho^{\prime \prime}\right]$ where $\rho^{\prime}$ is a trial density. The computed value $\Delta E_{\mathrm{D}}$ " is the trial density analog of $\Delta E_{\mathrm{D}}$ ' and equals $\Delta E_{\mathrm{D}}$ ' in the case where $\rho^{\prime}=\rho_{\text {exact. }}$ I refer to this number as $\Delta E_{\mathrm{D}}{ }^{\prime \prime}$ in this paper to signify that it was estimated from trial densities. I define "practically normal" systems as those for which a diverse trial set of functionals produce an average $\Delta E_{D}{ }^{\prime \prime}<4 \mathrm{~kJ} / \mathrm{mol}$ (chemical accuracy).

The differences in $\rho$ obtained for different pairs of the four functionals for 12 of the electronic systems studied by Medvedev et al. ${ }^{2}$ are shown in Supporting Information, Figures S2-S13 along the x-axis in units of bohr. The main differences between densities produced by these four diverse functionals are located near the nucleus $(x=0)$. Notably, PBE and B3LYP produce the most similar $\rho$, as shown by the yellow lines, but start to deviate at large $Z$ (compact density limit). The $\rho$ produced by the local spin density approximation SVWN deviates the most from the other functionals (red, blue, and gray colors) for all 12 systems. However, M06 is also quite distinct from B3LYP and PBE in most cases, and interestingly, the $\Delta \rho$ of B3LYP-M06 and PBE-M06 displays non-monotonic behavior near the nucleus (i.e. the difference is largest at the nucleus but then has a second maximum deviation at $+/-0.1$ bohr. This second maximum is 
reduced as $Z$ increases. The deviation in $\rho(\mathrm{SVWN})$ grows monotonically with $Z$ as $\rho$ becomes more compact and the lack of gradient manifests around the nucleus (the nuclear cusp condition implies that the change in $\rho$ at the nucleus scales linearly with $Z)^{34}$. Accordingly, in general, all errors in densities for the $1 s^{2} 2 s^{2}$ systems that dominate the total error in the previous study scale almost linearly with $Z^{10}$. The difference between M06 and B3LYP/PBE is generally larger for $1 \mathrm{~s}^{2}$ systems than for $1 \mathrm{~s}^{2} 2 \mathrm{~s}^{2}$ systems.

Figure 1B shows a numerical example for the $\mathrm{B}^{3+}$ ion, which is one of the cations studied previously $^{2}$, using PBE and SVWN. For this system, as well as other systems (see numerical data in Supporting Information), while the absolute differences in $E_{\mathrm{el}}$ are large and not meaningful on the non-variational "functional axis", the numbers along the vertical variational axis corresponding to $\Delta E_{\mathrm{D}}$ " are $\sim 3 \mathrm{~kJ} / \mathrm{mol}$. Due to the variational principle applied to densities ${ }^{4}$, the native combination produces smaller absolute energies. This is exemplified in Figure 1B from the signs +3 and $-3 \mathrm{~kJ} / \mathrm{mol}$ when computing energies according to a thermodynamic cycle, where the energy of change equals the energy of the end state minus the energy of the start state. Despite the major differences in these functionals, the error is already small in the absolute (nonobservable) $E_{\mathrm{el}}$.

For the ions studied previously ${ }^{2}$, the quality of $\mathrm{E}[\rho]$ can be estimated by computing the energy of removing the two 2 s electrons, e.g. $\mathrm{E}\left(\mathrm{B}^{3+}\right)-\mathrm{E}\left(\mathrm{B}^{+}\right)$, which can be compared directly to accurate experimental data from NIST $^{10}$. As seen in Figure 1C, for this energy, errors using a trial density reduce in both directions to $0.7 \mathrm{~kJ} / \mathrm{mol}$. The values of these energies are many tens of $\mathrm{eV}$, as discussed previously ${ }^{10}$. In the boron case, the experimental number is $63.1 \mathrm{eV}$. The $\rho$ derived error using SVWN as trial density instead for PBE is $0.7 \mathrm{~kJ} / \mathrm{mol}$ or about $0.01 \%$ of the total computed number, and $\sim 4 \%$ of the total error that PBE makes $(18.3 \mathrm{~kJ} / \mathrm{mol}$ error $)$. The four 
values are $\mathrm{SVWN}(\mathrm{SVWN})=6025.7 \mathrm{~kJ} / \mathrm{mol}, \mathrm{SVWN}(\mathrm{PBE})=6025.0 \mathrm{~kJ} / \mathrm{mol}, \mathrm{PBE}(\mathrm{PBE})=6068.7$ $\mathrm{kJ} / \mathrm{mol}$, and $\mathrm{PBE}(\mathrm{SVWN})=6067.9 \mathrm{~kJ} / \mathrm{mol}$ (the experimental number is $6086.9 \mathrm{~kJ} / \mathrm{mol}$ ).

Since the difference between the experimental value and PBE is only a third of the difference between experiment and SVWN, and considering the $\rho$ improvements using PBE vs. SVWN (SI, Figures S2-S13 and previous work ${ }^{2}$ ), we could use the jargon ${ }^{2}$ that PBE is more "exact" for $\mathrm{B}^{+}$and $\mathrm{B}^{3+}$ than SVWN, and roughly speaking two-thirds of the way towards “exactness" from SVWN for this system. From these distance-metric considerations, we can argue that $\Delta \mathrm{E}_{\mathrm{D}}{ }^{\prime}$ will continue to be very small compared to $\Delta E_{\mathrm{F}}{ }^{\prime}$ also in the final step towards exactness, although we do not know the exact $E[\rho]$ for the boron ions (or any other system).

The results in Figure 1C suggest that trial densities such as PBE may sometimes be used to search for exact functionals, because the error made in $\rho$ is quite small, consistent with previous suggestions ${ }^{11}$. Systems such as $\mathrm{B}^{+}$and $\mathrm{B}^{3+}$ will have $\Delta E_{\mathrm{D}}$ ' so small that it becomes insignificant, i.e. any optimization of $\rho$ reduces $\Delta E_{\mathrm{D}}{ }^{\prime}$ from $1 \mathrm{~kJ} / \mathrm{mol}$ to a smaller number within the uncertainty of other approximations of the non-relativistic Born-Oppenheimer-type KohnSham calculation.

Because $\Delta E_{\mathrm{D}}$ ' of a calculation depends both on system and functional, functionals may also be defined as normal or abnormal. I define here "practically normal" as a functional and a system (external potential) that together give $\Delta E_{\mathrm{D}}{ }^{\prime}<4 \mathrm{~kJ} / \mathrm{mol}$ (chemical accuracy). Thus, if $a$ calculation gives $\Delta E_{D}^{\prime}<4 \mathrm{~kJ} / \mathrm{mol}$ for a suitable diverse test set of functionals, the system and the functional are normal. Again, we can search for the exact functional for normal systems using fixed trial densities to within chemical accuracy, making the approximation $\Delta E_{D}^{\prime} \sim \Delta E_{D}{ }^{\prime \prime}$. In contrast, universality will only be effectively approached by studying abnormal systems where the energy functional changes more substantially with variations in $\rho^{11}$. 
Similar computations were done for all combinations of the trial test set SVWN, B3LYP, PBE, and M06, with all the cations $\mathrm{B}^{+}, \mathrm{B}^{3+}, \mathrm{C}^{2+}, \mathrm{C}^{4+}, \mathrm{N}^{3+}, \mathrm{N}^{5+}, \mathrm{O}^{4+}, \mathrm{O}^{6+}, \mathrm{F}^{5+}, \mathrm{F}^{7+}, \mathrm{Ne}^{6+}$, and $\mathrm{Ne}^{8+}$, as studied previously ${ }^{2,10}$ (see Supporting Information Tables S1-S6 for numerical details). To make data more accessible, the average of the deviations in double IPs caused by the tree other trial densities were computed for all six cases; these average values represent sensitivities of the computed observable energy to the use of trial densities and are shown for all four functionals in Figure 2A (for B3LYP), Figure 2B (for PBE), Figure 2C (for SVWN), and Figure 2D (for M06).

From Figure 2, it can be seen that the effect of using a trial density on the numerically very large energy of removing two 2 s electrons $(43-446 \mathrm{eV}$ experimentally) is maximally 3 kJ/mol. M06 produces the "worst" trial densities for B3LYP and SVWN (Figure 2A, C), and that B3LYP and PBE provide uniformly accurate trial densities for each other. In fact, instead of computing the more expensive $E_{\mathrm{B} 3 \mathrm{LY}}\left(\rho_{\mathrm{B} 3 \mathrm{LYP}}\right)$, one may compute $E_{\mathrm{B} 3 \mathrm{LY}}\left(\rho_{\mathrm{PBE}}\right)$ by a single energy calculation on the frozen, previously optimized (without expensive Hartree-Fock exchange) $\rho_{\mathrm{PBE}}$ and obtain results that differ from the fully optimized B3LYP values by $<1 / 2 \mathrm{~kJ} / \mathrm{mol}$ in all six cases (Figure 2A). This confirms the suggestion ${ }^{10}$ that the errors in $\rho$ of these systems ${ }^{2}$ are not numerically relevant and thus "normal" in the terminology of Burke and co-workers ${ }^{11}$.

The density differences plotted in Figures S2-S13 agree well with the energetics of Figure 2; thus, B3LYP/PBE are the most similar behaving functionals and can be used with high confidence as trial densities for each other for these systems. Interestingly, these differences provide a semi-quantitative metric of the similarity of functionals that is consistent both in $\rho$ and $E[\rho]$ space and thus may in principle be used as a quantification of degree of exactness, if a similar metric was applied to the exact functional, which is of course unknown. 

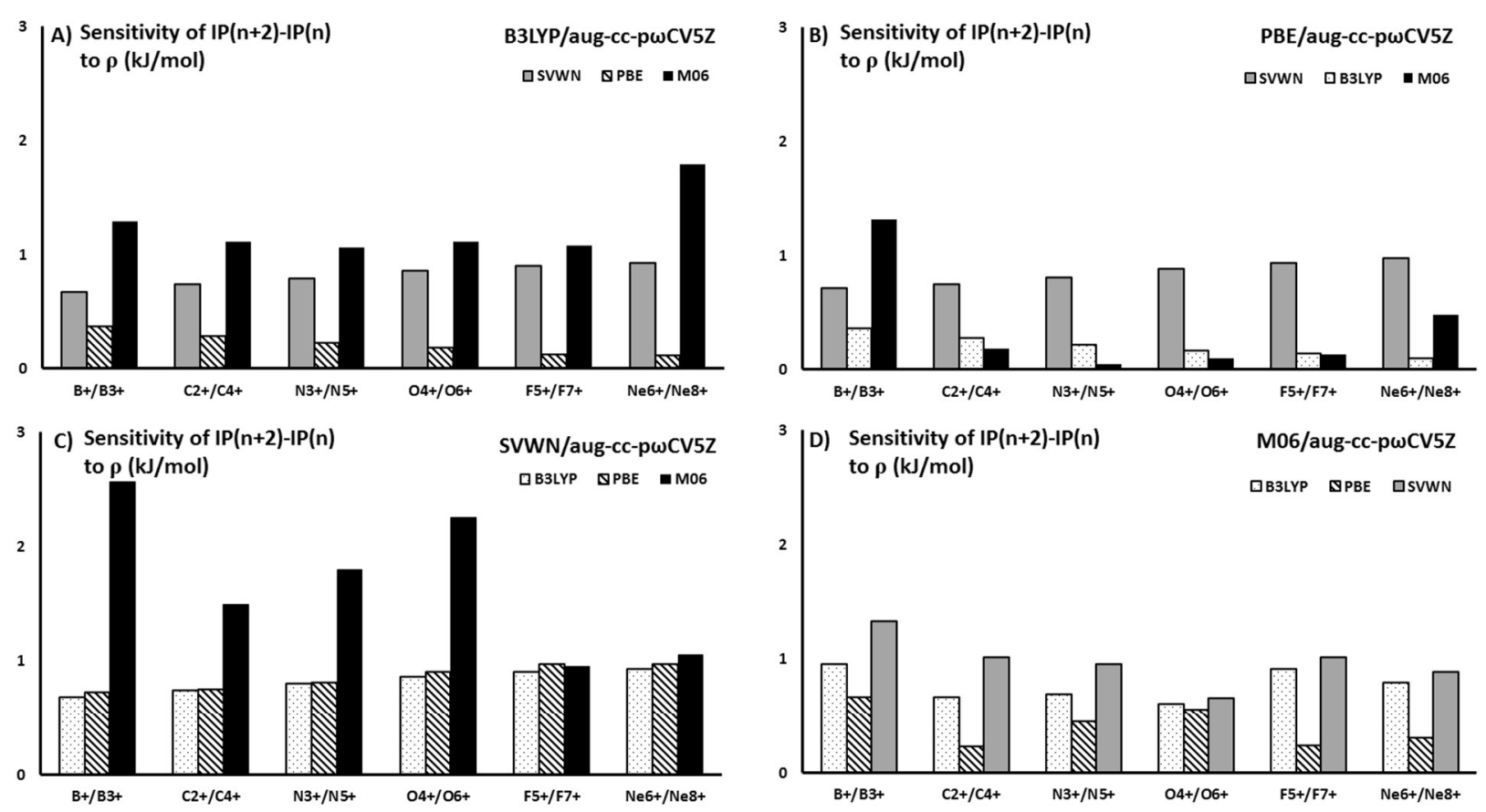

Figure 2. Mean absolute deviation between double IPs computed with the native $E[\rho]$ and using trial densities E[ $\left.\rho^{\prime}\right]$. A) B3LYP energies using SVWN, PBE, and M06 for $\rho^{\prime}$. B) PBE energies using SVWN, B3LYP, and M06 for $\rho^{\prime}$. C) SVWN energies using B3LYP, PBE, and M06 for $\rho^{\prime}$. D) M06 energies using SVWN, B3LYP, and PBE for $\rho^{\prime}$.

To confirm that the trial set is estimates $\Delta E_{\mathrm{D}}$ " well, the average $\Delta E_{\mathrm{D}}$ " for the double IPs of $\mathrm{B}^{+}, \mathrm{B}^{3+}, \mathrm{C}^{2+}, \mathrm{C}^{4+}, \mathrm{N}^{3+}, \mathrm{N}^{5+}, \mathrm{O}^{4+}, \mathrm{O}^{6+}, \mathrm{F}^{5+}, \mathrm{F}^{7+}, \mathrm{Ne}^{6+}$, and $\mathrm{Ne}^{8+}$ were also compared with another trial set involving the more diverse (in terms of previously reported errors in densities) functionals M06-2X, PBE0, PBE, and SVWN. This set represents largest differences in errors of the densities and are compared to the M06-PBE-SVWN-B3LYP trial test set in Figure S14. As can be seen, the average energy effects of interchanging these densities remain $<2 \mathrm{~kJ} / \mathrm{mol}$, confirming the conclusion above.

The effects described here arise from the differential behavior of the exchange correlation potentials and are exemplified by $\mathrm{PBE}$ applied to $\mathrm{B}^{+}$and $\mathrm{Ne}^{6+}$ in Figure 3A-B and Figure C-D, 
respectively. When the exchange correlation potential of the native density (Figure $\mathbf{3 A}$ and Figure 3C) is evaluated using a trial density, the differences in densities translate into an effect on the functional's exchange-correlation potential (Figure 3B and Figure 3D), which then translates into energy. It can be seen that whereas the absolute potentials are of course localized near the nuclei, the differential effects central to the discussion of the $\rho$-variations spread over much of the electronic system, explaining why these energy effects do not increase monotonically with nuclear charge and compactness of the electron density as the errors in $\rho$ themselves do for these systems.

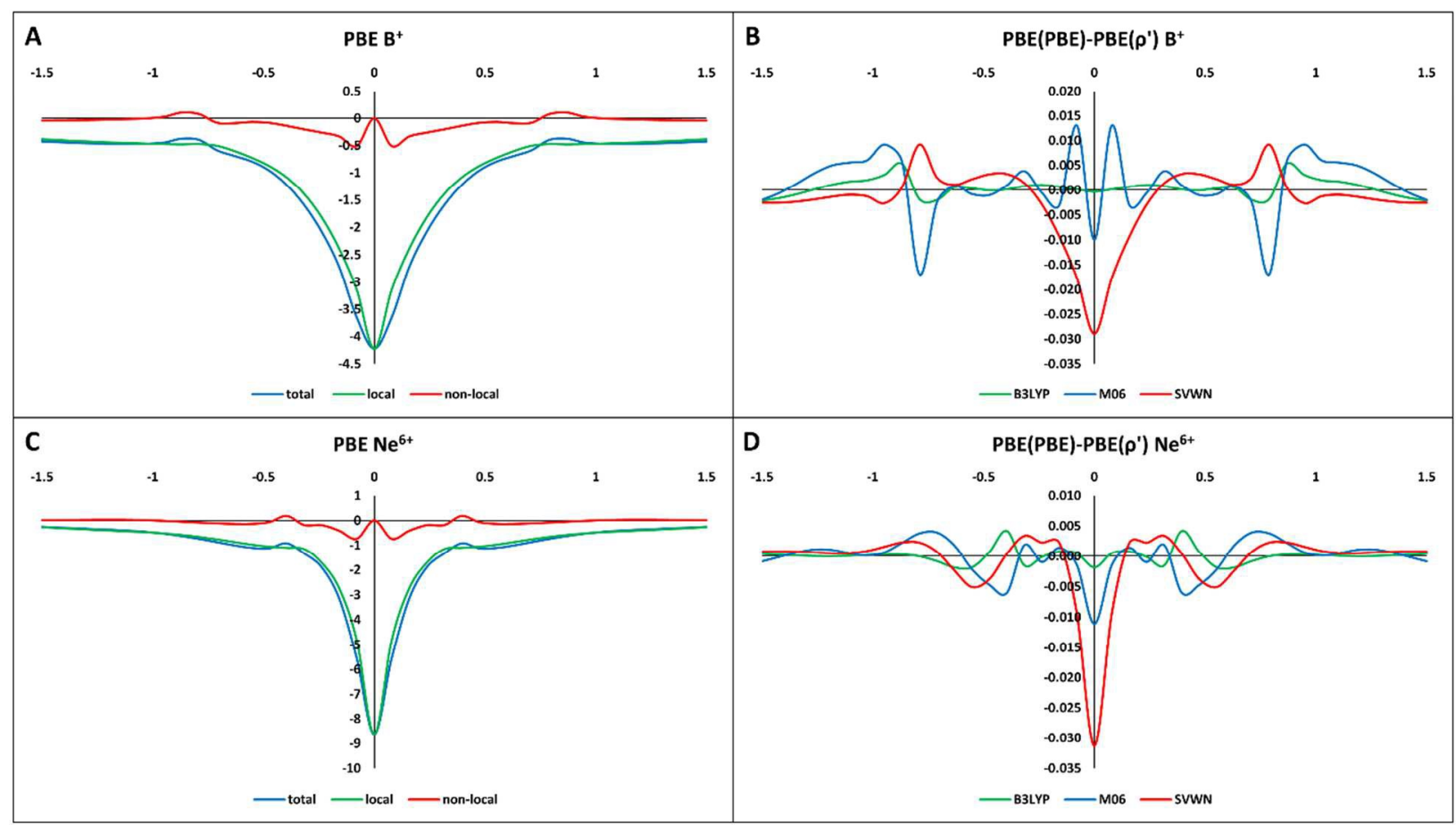

Figure 3. A) PBE exchange-correlation potential for $\mathrm{B}^{+}$applied to the native PBE density; B) same but applied to three other trial densities converged with other functionals; C) PBE exchange-correlation potential for $\mathrm{Ne}^{6+}$ applied to the native PBE density; D) same but applied to three other trial densities converged with other functionals. 
First Ionization Potentials of Neutral Atoms. In the following, the above procedure is applied to the study of the more chemically relevant first IPs of atoms. To avoid the complication of relativistic effects, only the first and second period of the periodic table were studied. The experimental data are in Table S7; Tables S8-S10 show numerical details for native method combinations, whereas Tables S11-S14 show results using trial densities. Table 1 shows the computed IPs using the aug-cc-pV5Z basis set and the methods CCSD(T), CCSD, B3LYP, SVWN, PBE, and M06. For these experimentally observable energies, CCSD(T) achieves a mean absolute error (MAE) of $\sim 1.5 \mathrm{~kJ} / \mathrm{mol}$, i.e. the description of the systems is essentially correct to within this limit (scalar relativistic effects are $<1 \mathrm{~kJ} / \mathrm{mol}$ for these energies ${ }^{10}$ ). Among the four functionals, M06 displays the smallest MAE, probably because it was parameterized to fit these data ${ }^{31}$. The main question of interest here is how much $\rho$ contributes to the energy errors, because this should be required of a good density norm.

Table 1. Errors in computed ionization potentials (aug-cc-pV5Z basis, in $\mathrm{kJ} / \mathrm{mol}$ ).

\begin{tabular}{|l|l|l|l|l|l|l|}
\hline & CCSD(T) & CCSD & B3LYP & PBE & SVWN & M06 \\
\hline $\mathrm{H}$ & 0.49 & 0.49 & -1.99 & 0.46 & -55.50 & 1.00 \\
\hline $\mathrm{He}$ & -1.19 & -1.19 & 24.93 & -11.94 & -27.75 & 18.22 \\
\hline $\mathrm{Li}$ & -1.15 & -1.23 & 15.14 & 18.69 & 7.81 & -8.24 \\
\hline $\mathrm{Be}$ & -1.41 & -2.81 & -28.58 & -31.58 & -28.70 & -37.39 \\
\hline $\mathrm{B}$ & -1.75 & -5.12 & 34.62 & 36.27 & 33.72 & 13.09 \\
\hline $\mathrm{C}$ & -1.04 & -3.80 & 18.81 & 26.93 & 41.59 & 5.72 \\
\hline $\mathrm{N}$ & 0.04 & -2.70 & 3.25 & 18.21 & 45.07 & 19.40 \\
\hline $\mathrm{O}$ & -4.75 & -10.08 & 39.76 & 42.58 & 37.02 & 18.43 \\
\hline $\mathrm{F}$ & -2.58 & -9.11 & 18.65 & 21.95 & 51.31 & 11.87 \\
\hline $\mathrm{Ne}$ & 1.15 & -6.32 & 3.29 & 8.28 & 59.54 & 9.95 \\
\hline $\boldsymbol{M} \boldsymbol{A} \boldsymbol{E}$ & $\mathbf{1 . 5 5}$ & $\mathbf{4 . 2 8}$ & $\mathbf{1 8 . 9 0}$ & $\mathbf{2 1 . 6 9}$ & $\mathbf{3 8 . 8 0}$ & $\mathbf{1 4 . 3 3}$ \\
\hline
\end{tabular}


Figure 4A and $4 \mathrm{~B}$ show the differential electron densities for two of these more electronrich systems, $\mathrm{Ne}$, and $\mathrm{Ne}^{+}$. It is notable that the same patterns in the differential densities observed for the closed-shell s-configurations still hold for the more electron rich pconfigurations, including the open-shell systems, exemplified by $\mathrm{Ne}^{+}$(Figure 4B).

Figure 4C and 4D show the average sensitivity (mean absolute deviation between energies obtained using the native density and trial densities) using the aug-cc-pV5Z and defTZVP basis sets, respectively (Figure S15 shows the full plots without averaging. The data with def-TZVP are in Tables S15 and S16). The errors obtained with each native functional and density combination are given in Table S17 for both basis sets. As seen from Figures $\mathbf{4 C}$ and 4D, with both basis sets, the average effect of the trial densities on computed IPs is smaller than $2 \mathrm{~kJ} / \mathrm{mol}$ except for $\mathrm{Ne}$, where M06 deviates on average by $4 \mathrm{~kJ} / \mathrm{mol}$, more than any other functional including SVWN, although its densities are more similar to those of B3LYP and PBE. This relates to the variation of the exchange-correlation energy with density, i.e. to the exchange correlation potential functional, $v_{\mathrm{xc}}=\partial \mathrm{E} / \partial \rho$, e.g. Figure 3B. The meta feature and heavy parameterization of M06 could affect the smoothness of its electron density near the nucleus where the energy and density effects are most important. This is a general tendency despite the distinct s- and p-electronic configurations and number of unpaired electrons, and regardless of basis set used (hydrogen and beryllium are exceptions). It is also interesting to observe that M06 is approximately doubly as basis-set sensitive as B3LYP (1.89 vs. $0.99 \mathrm{~kJ} / \mathrm{mol}$ average, Table S18, for def-TZVP vs. aug-cc-pV5Z) and also more than doubly as sensitive to $\rho$ as B3LYP (1.01 vs. $0.37 \mathrm{~kJ} / \mathrm{mol}$ ). In order words, it suggests that basis-set and density sensitivity are correlated. The basis set sensitivity and the density sensitivity probably both arise from the same underlying behavior of the exchange correlation potential (Figures 3B, 3D), and e.g. smoothness constraints may improve M06 in this regard. 

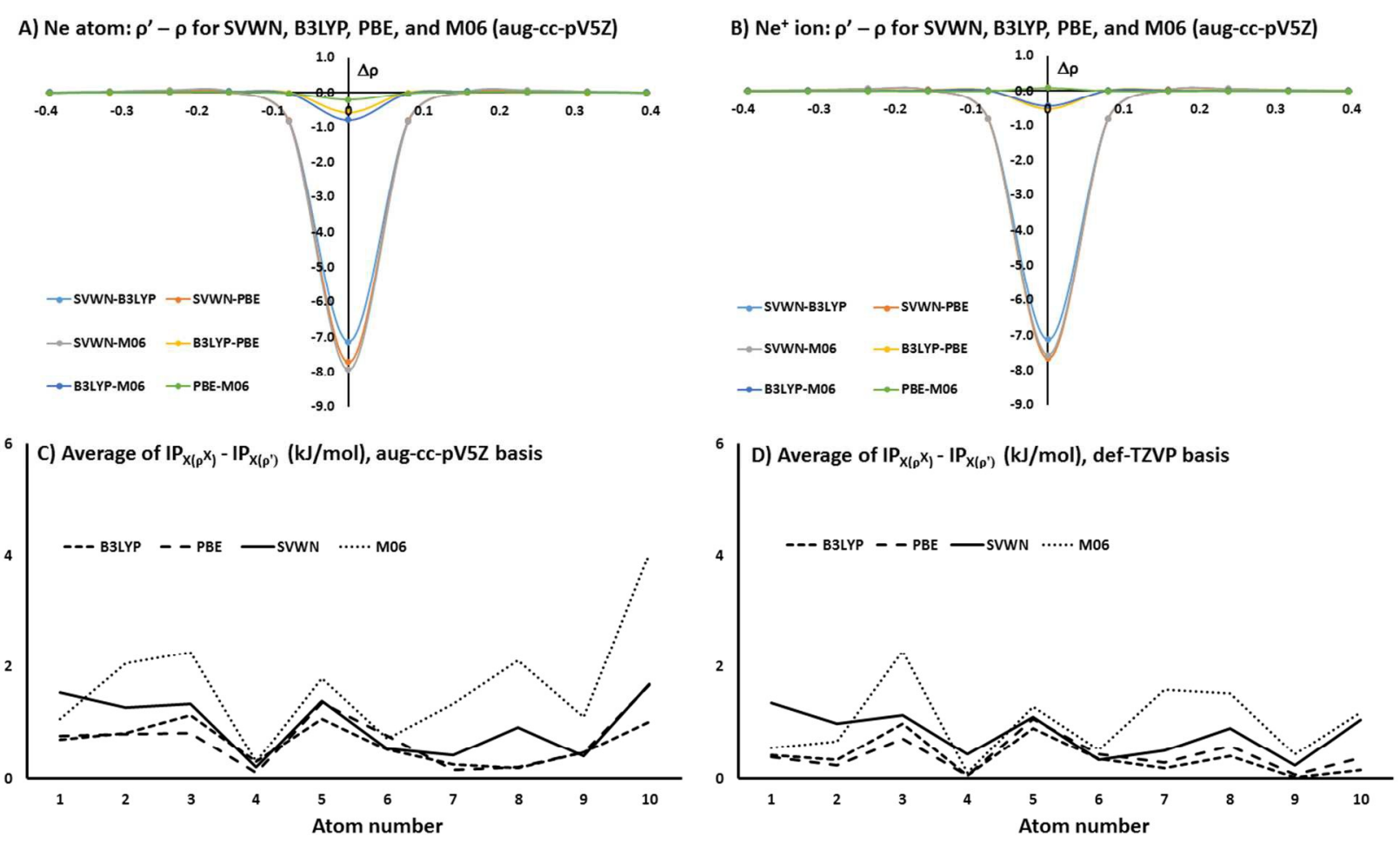

Figure 4. A) Differential electron densities $\rho-\rho^{\prime}$ for $\mathrm{Ne}$ atom; B) same for $\mathrm{Ne}^{+}$ion; C) average sensitivity of four functionals to the use of trial $\rho^{\prime}$ from other functionals at the aug-cc-pV5Z basis set level; D) same at the def-TZVP basis set level.

Another relevant observation is an oscillating behavior in the sensitivity toward $\rho$ ', which reflects variable (practical) normality of the electronic systems; thus, $Z=1,4,7$, and 9 show the highest "normality", whereas $Z=3,5,8$, and 10 are more "abnormal". These differences can be traced to the difficulty of describing $\rho$ when electronic configurations change qualitatively upon ionization (simply speaking, in terms of their n, 1, and s quantum numbers). Thus, Li goes from the high-energy delocalized $2 \mathrm{~s}$ to a fully symmetric closed-shell $1 \mathrm{~s}^{2}$ configuration in $\mathrm{Li}^{+}$. B goes from an open-shell $2 \mathrm{p}^{1}$ configuration to a fully symmetric closed-shell $1 \mathrm{~s}^{2} 2 \mathrm{~s}^{2} \mathrm{~B}^{+}$state. $\mathrm{O}$ has to remove a paired p-electron from $[\mathrm{He}] 2 \mathrm{~s}^{2} 2 \mathrm{p}^{4}$ to produce a particularly stable maximum-spin, halfoccupied p-shell (for $\mathrm{N}$, p-orbital pairing is avoided; in $\mathrm{F}$, there are two paired p-orbitals so the 
effect on pairing is relatively smaller). Ne goes from a completely filled closed shell to a delocalized open-shell hole in the 2 p-shell. Regardless of this, all these systems are practically normal if we use chemical accuracy $(4 \mathrm{~kJ} / \mathrm{mol})$ as a threshold.

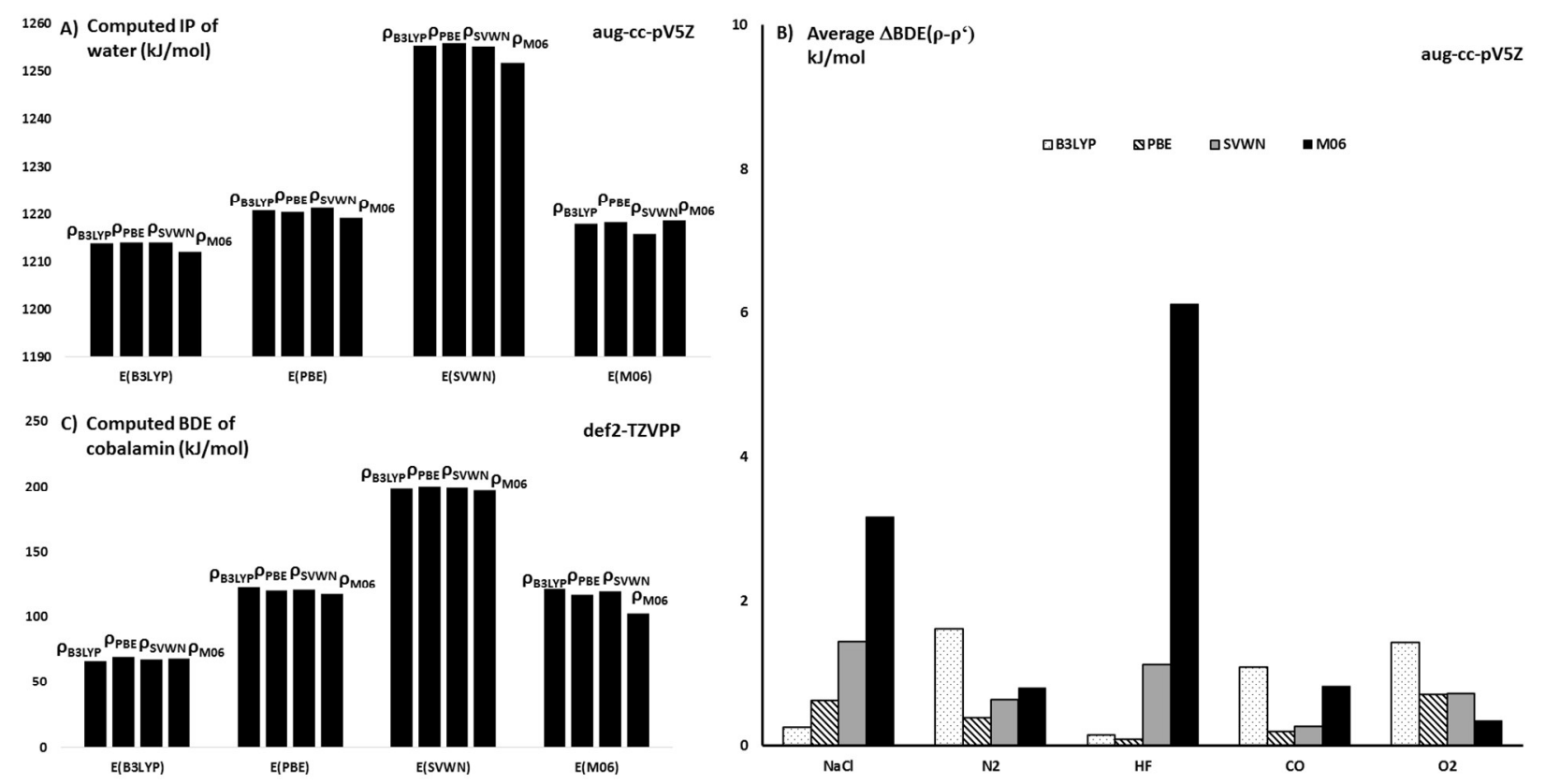

Figure 5. A) Computed ionization potentials (aug-cc-pV5Z) of $\mathrm{H}_{2} \mathrm{O}$ using all 16 trial combinations of B3LYP, PBE, SVWN, and M06. B) Average change in computed BDE of $\mathrm{NaCl}, \mathrm{N}_{2}, \mathrm{HF}, \mathrm{CO}$, and $\mathrm{O}_{2}$ when using other densities than the native density, for B3LYP, PBE, SVWN, and M06 and the aug-cc-pV5Z basis set. C) Computed Co-C BDE of 5'deoxyadenosylcobalamin at the def2-TZVPP basis set level, using all 16 possible trial combinations.

Examples for Molecular Systems. To illustrate the procedure on molecular systems, three more cases were studied: The ionization potential of the water molecule, computed BDE of diatomic molecules, and the Co-C BDE of the large coenzyme 5'-deoxyadenosylcobalamin, one 
of the active forms of vitamin $\mathrm{B}_{12}$. The data set of diatomic molecules represents ionic bonding $(\mathrm{NaCl}, \mathrm{HF})$, covalent bonding $\left(\mathrm{N}_{2}, \mathrm{O}_{2}\right)$, and polar covalent bonding $(\mathrm{CO})$ as well as single $(\mathrm{HF}$, $\mathrm{NaCl})$, double $\left(\mathrm{O}_{2}\right)$, and triple bond $\left(\mathrm{N}_{2}, \mathrm{CO}\right)$ character. The variations of these computed observables with changes in $\rho^{\prime}$ are summarized in Figure 5.

Figure 5A shows the IP computed for the water molecule using any of the 16 combinations of density functionals and densities. The computed electronic energies are described in detail in the Supporting Information Tables S19-S23. The experimental IP is 1220.6 $\mathrm{kJ} / \mathrm{mol}$. The computed values using B3LYP, PBE, SVWN, and M06 are 1213.8, 1220.5, 1255.2, and $1218.8 \mathrm{~kJ} / \mathrm{mol}$, respectively. Computing the IP using trial densities produce similar IP values to within $4 \mathrm{~kJ} / \mathrm{mol}$; the largest variation is seen for the local SVWN functional. Again, the PBE and B3LYP functionals give results within $1 \mathrm{~kJ} / \mathrm{mol}$ when using the other as trial density, and M06 is somewhat more sensitive than these two to the nature of $\rho^{\prime}$.

Figure 5B show the average change in the $\mathrm{BDE}$ of $\mathrm{NaCl}, \mathrm{N}_{2}, \mathrm{HF}, \mathrm{CO}$, and $\mathrm{O}_{2}$ for each functional using its own functional vs. using the other densities as trial densities, referred to as $\triangle \operatorname{BDE}\left(\rho-\rho^{\prime}\right)$, all computed with the aug-cc-pV5Z basis set for the five diatomic molecules. A small $\triangle B D E\left(\rho-\rho^{\prime}\right)$ implies that the functional is not very sensitive to the use of trial density for the particular electronic system. The BDEs for the five molecules were also computed with CCSD(T)/aug-cc-pV5Z and CCSD/aug-cc-pV5Z including ZPE (Table S24) to estimate accuracy of the densities produced from this basis set. A mean absolute error (MAE) for $\operatorname{CCSD}(\mathrm{T})$ of $5.3 \mathrm{~kJ} / \mathrm{mol}$ is obtained using the aug-cc-pV5Z basis set (Table S25). Part of the error in $\operatorname{CCSD}(\mathrm{T})$ relates to relativistic corrections (of the order of $\sim 1 \mathrm{~kJ} / \mathrm{mol}$, estimated from scalar-relativistic contributions, and enthalpy corrections (numerical results in Tables S26-S27 for native methods and in Tables S28-S31 using trial densities). 
For these systems, B3LYP and PBE produce small variations in BDE when $\rho$ ' is varied. It confirms that when studying also "normal" molecular systems, one can use any reasonably accurate $\rho$ and save substantial computer time. However, in the two cases of strong ionic bonding $(\mathrm{NaCl}$ and $\mathrm{HF}) \rho_{\mathrm{M} 06}$ displays an average sensitivity of $\sim 6 \mathrm{~kJ} / \mathrm{mol}$, even though the electronic systems are practically normal. Again this reflects that M06 deviates the most from the other functionals near the energy-wise important nuclei. As seen, "normality" of the systems is not directly related to the strength of the bonds (the dense electron limit) i.e. all these systems are practically normal despite their distinct bonding behaviors, yet are important benchmarks for describing accurate thermochemistry. Abnormal systems may be diffuse or delocalized densities, e.g. anions or dissociating states, as discussed previously ${ }^{11}$. The use of orbital-dependent HF densities as "trial" densities, $\Delta E\left[\rho^{\prime}\right]$ where $\rho^{\prime}=\rho_{\mathrm{HF}}$, is often much more accurate for such abnormal systems ${ }^{20}$, where the self-interaction error also manifests.

As a final example of a large system, Figure 5C shows the computed Co-C BDEs for 5'deoxyadenosylcobalamin, the active form of vitamin $\mathrm{B}_{12}$, with 676 electrons. For such large systems, accurate thermochemistry depends critically on the inclusion of dispersion corrections and solvent effects ${ }^{22}$; the Cosmo model $^{23}$ for water was used as well as D3 corrections ${ }^{25}$, except for SVWN. As can be seen from Figure 5C (numerical data in Table S32), the Co-C BDEs are generally not very sensitive to the used $\rho^{\prime}$ even for this large, complex electronic system. As described previously, for this particular system, PBE gives more accurate results (the experimental value is $\sim 130 \mathrm{~kJ} / \mathrm{mol})^{22}$. It is surprising that a good estimate is obtained regardless of the $\rho^{\prime}$ used with PBE, implying that even a challenging problem like the Co-C BDE of vitamin $\mathrm{B}_{12}$ belong to the class of "normal" systems. Consequently, we can obtain the Co-C BDE of any normal functional very quickly to using any normal, previously converged density such as $\rho_{\mathrm{PBE}}$, making errors $<4 \mathrm{~kJ} / \mathrm{mol}$ (chemical accuracy); the single-point energy evaluations on fixed 
densities are multi-fold faster than a fully converged computation. For example, the total cpu time for converging the full density and energy for the full system was $17 \mathrm{~h} 51 \mathrm{~m}$ with B3LYP, but only 17 minutes for the subsequent PBE calculation. The full PBE convergence took $3 \mathrm{~h} 39 \mathrm{~m}$, and the B3LYP calculation using this density $4 \mathrm{~h} 27 \mathrm{~m}$ (very similar, $4 \mathrm{~h} 31 \mathrm{~m}$ if using the M06 density). Thus in particular for non-hybrid functionals that do not require evaluation of the exchange integrals, the saved computer time is a factor of ten for this system, losing an accuracy of 3-4 kJ/mol. Consequently, even though B3LYP fails substantially in describing this BDE, as discussed in the literature ${ }^{35,36}$, PBE performs very well even on the B3LYP density; in other words, in the "thermodynamic cycle" of PBE and B3LYP, the change in $\rho$ affects the energy 16 times less than the change in functional. M06 stands out by having the largest sensitivity to trial density by a factor of $2(8.3 \mathrm{~kJ} / \mathrm{mol}$ vs. 1.2-3.9 kJ/mol for the other three, Table S32), again confirming its more sensitive exchange correlation potential vs. the other functionals, a feature that also seems to make it more basis-set dependent.

\section{Conclusions.}

In the search for more exact and generally applicable density functionals, the lack of systematic recipes of improvement and of well-defined "paths" toward exactness is a major challenge. As discussed in this work, the Hohenberg-Kohn theorems are central to this challenge. Dividing errors in a functional into a term for $\rho\left(\Delta E_{\mathrm{D}}{ }^{\prime}\right)$ and one for the effect of the energy functional on $\rho$ $\left(\Delta E_{\mathrm{F}}^{\prime}\right)$ (including manipulations of its gradient, Laplacian, etc.), as recently suggested by Burke and co-workers ${ }^{11}$, enables a formal strategy for improving functionals, as outlined in Figure 1. Specifically, the direct path toward exactness from any functional is defined here as the straight diagonal line towards the upper right in Figure 1A: This path is defined by $\Delta E_{\mathrm{D}}{ }^{\prime} \rightarrow 0$ and $\Delta E_{\mathrm{F}}{ }^{\prime}$ 
$\rightarrow 0$ by the same scale factor of the total error in $E^{\prime}\left[\rho^{\prime}\right]$ as $\rho^{\prime} \rightarrow \rho_{\text {exact }}$ and $E^{\prime}\left[\rho^{\prime}\right] \rightarrow E_{\text {exact }}\left[\rho_{\text {exact }}\right]$. "Straying" then becomes well-defined as any change in a functional that increases $\Delta E_{\mathrm{D}}{ }^{\prime}$ or $\Delta E_{\mathrm{F}}$ '.

To determine deviation from the path, we must separate energy effects due to $\rho$ and due to the way $\rho$ is converted into a property of interest (here: $E[\rho]$ ) by the functional. Unfortunately, the exact $\Delta E_{\mathrm{D}}{ }^{\prime}$ suggested by Burke et al. ${ }^{11}$ (i.e. compared to exact densities and energies) is generally inaccessible, preventing its use in estimating deviations from the path defined in Figure 1A for most electronic systems of interest. To solve this problem, trial densities $\rho^{\prime}$ for a suitably chosen trial test set of functionals can be used. These should i) be small (4-5 functionals) to make sensitivity analysis computationally tractable; ii) consist of diverse functionals, preferably from different rungs of Jacob's ladder, to estimate as broadly as possible the density sensitivity. The present analysis shows that systems and functionals can both be classified as "practically normal" or abnormal via their average $\Delta E_{\mathrm{D}}$ " for the defined trial test set. I define here a practically normal calculation as one that has $\Delta E_{\mathrm{D}}{ }^{\prime \prime}<4 \mathrm{~kJ} / \mathrm{mol}$ (chemical accuracy).

Using the protocol and definitions, a range of electronic systems are studied, starting with the systems previously studied by Medvedev et al. ${ }^{2}$ because they specifically suggested a norm for densities, but then moving to more chemically relevant systems. Additional conclusions arising from these calculations are:

1) Variations in $E\left[\rho^{\prime}\right]$ using a suitably diverse test set of trial densities offers a fast and efficient way to estimate the sensitivity of the energy to variations in $\rho$; this enables a fast way to estimate if variations in $\rho$ have measurable effects on $E[\rho]$, i.e. if they are useful norms for assessing functional performance or are within the noise of computational chemistry.

2) Previously studied ${ }^{2}$ compact atomic ions used to conclude that density functionals are straying are "practically normal" in the sense that $\Delta E_{\mathrm{D}}$ " $<4 \mathrm{~kJ} / \mathrm{mol}$. Thus, this straying is not 
numerically significant vs. other approximations in the Kohn-Sham procedure, such as the use of Gaussian basis functions for very compact electron densities, whose main differences manifest near the nuclei. For example, IPs produced by PBE and B3LYP using each other's densities are typically $\sim 1 \mathrm{~kJ} / \mathrm{mol}$. The protocol thus rules out these systems as good norms.

3) More chemically relevant atoms and monocations for the first and second row $(Z=1$ 10) studied in this work, and accordingly also first ionization energies, are also practically normal, and the energy consequences of varying densities are thus too small to be chemically relevant and mostly beyond assessment of accuracy considering other approximations done in the Kohn-Sham Born-Oppenheimer formalism that could affect energies up to a few $\mathrm{kJ} / \mathrm{mol}$.

4) Bond dissociation energies of diatomic molecules are practically normal for large basis sets, but can become less normal for large systems or smaller basis sets; thus while B3LYP and PBE give very similar results for the Co-C BDE of the large complex 5'deoxyadenosylcobalamin if the other's $\rho$ ' is used for calculation, M06 gives very different results depending on $\rho^{\prime}$, probably because this functional has a more sensitive exchange correlation functional, viz. Figure 4. Still however, even large electronic systems such as the cobalamins can be studied with simpler trial densities to speed up single point computations by loss of only a few $\mathrm{kJ} / \mathrm{mol}$ in accuracy (e.g. using B3LYP with PBE trial densities).

This analysis may be useful in focusing efforts towards developing new functionals. For example, $\rho^{\prime}(\mathrm{PBE})$ will serve excellently as trial density in the search for $\rho$ if the benchmarked electronic systems are normal (e.g. IPs and BDEs computed by the separation method ${ }^{11}$ ); but to move further, one needs to improve functionals for abnormal systems where errors in $\rho^{\prime}$ become significant vs. chemical accuracy. For errors smaller than this, it is hardly testable whether this contributes to exactness or not, because the uncertainty resembles that of other approximations 
tacitly applied. Therefore, an absolute definition of exact normality as $\Delta \mathrm{E}_{\mathrm{D}}{ }^{\prime}<4 \mathrm{~kJ} / \mathrm{mol}$ and practical normality as $\Delta \mathrm{E}_{\mathrm{D}}{ }^{\prime \prime}<4 \mathrm{~kJ} / \mathrm{mol}$ (chemical accuracy) seems reasonable.

Formal approaches to understand and improve density functional theory for use across the periodic table are desirable. As suggested here, this involves i) a separation of errors due to density and energy, as emphasized by Burke et al.; ii) a formal definition of paths toward

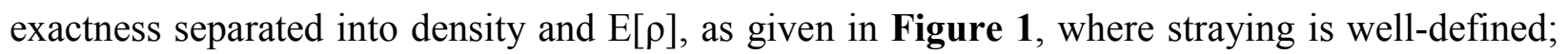
iii) a practical method to decompose errors along the two axes when exact data are unavailable, using sensitivity analysis of trial densities as explained in this paper. The resulting estimate of the sensitivity and precision of functionals applied to electronic systems may be useful for testing and comparing functionals because it enables a direct estimate of the significance of density variations in terms of energy. Specifically, emphasis should be on systems with $\Delta \mathrm{E}_{\mathrm{D}}{ }^{\prime}>4 \mathrm{~kJ} / \mathrm{mol}$, where density errors become meaningful and important to consider and improve.

Supplementary Information. The supplementary information file contains an example of the error in density due to the use of Gaussian functions (Figure S1), figures of differential density plots for the four functionals for $\mathrm{B}^{+}, \mathrm{B}^{3+}, \mathrm{C}^{2+}, \mathrm{C}^{4+}, \mathrm{N}^{3+}, \mathrm{N}^{5+}, \mathrm{O}^{4+}, \mathrm{O}^{6+}, \mathrm{F}^{5+}, \mathrm{F}^{7+}, \mathrm{Ne}^{6+}$, and $\mathrm{Ne}^{8+}$ (Figures S2-S13); average $\Delta E_{\mathrm{D}}$ " for the double IPs of $\mathrm{B}^{+}, \mathrm{B}^{3+}, \mathrm{C}^{2+}, \mathrm{C}^{4+}, \mathrm{N}^{3+}, \mathrm{N}^{5+}, \mathrm{O}^{4+}, \mathrm{O}^{6+}, \mathrm{F}^{5+}$, $\mathrm{F}^{7+}, \mathrm{Ne}^{6+}$, and $\mathrm{Ne}^{8+}$ using two different trial tests sets (Figure S14); plots of the difference in IPs obtained for individual functionals when using trial densities (Figure S15); electronic energies and double IPs for $\mathrm{B}^{+}, \mathrm{B}^{3+}, \mathrm{C}^{2+}, \mathrm{C}^{4+}, \mathrm{N}^{3+}, \mathrm{N}^{5+}, \mathrm{O}^{4+}, \mathrm{O}^{6+}, \mathrm{F}^{5+}, \mathrm{F}^{7+}, \mathrm{Ne}^{6+}$, and $\mathrm{Ne}^{8+}$ (Tables S1-S6); experimental IPs (Table S7); electronic energies and IPs of neutral atoms computed with all methods at aug-cc-pV5Z (Tables S8-S14) and def-TZVP (Tables S15-S16); errors in computed IPs (Table S17); basis set sensitivities of IPs (Table S18); data for the water molecule (Tables S19-S23); ZPEs (Table S24) and computed BDEs (Table S25) of diatomic molecules; computed 
electronic energies and $\mathrm{D}_{0}$ (without ZPE) of diatomic molecules (Tables S26-S31); computed Co-C BDE of 5'-deoxyadenosylcobalamin, and sensitivity to use of trial density (Table S32).

Acknowledgements. The Aarhus Supercomputer Center (formerly part of the Danish Center for Scientific Computing) is gratefully acknowledged for providing computer time for this project. 


\section{References}

1 J. Tao, J. P. Perdew, V. N. Staroverov and G. E. Scuseria, Phys. Rev. Lett., 2003, 91, 146401.

2 M. G. Medvedev, I. S. Bushmarinov, J. Sun, J. P. Perdew and K. A. Lyssenko, Science, 2017, 355, 49-52.

3 M. Levy, Proc. Natl. Acad. Sci., 1979, 76, 6062-6065.

$4 \quad$ P. Hohenberg and W. Kohn, Phys. Rev., 1964, 136, B864.

5 R. Peverati and D. G. Truhlar, Philos. Trans. R. Soc. London A Math. Phys. Eng. Sci., 2014, 372, 20120476.

6 T. Gould, J. Chem. Theory Comput., 2017, 13, 2373-2377.

7 R. G. Parr and Y. Weitao, Density-Functional Theory of Atoms and Molecules, Oxford University Press, 1994.

8 F. G. Cruz, K.-C. Lam and K. Burke, J. Phys. Chem. A, 1998, 102, 4911-4917.

9 K. P. Kepp, Coord. Chem. Rev., 2013, 257, 196-209.

10 K. P. Kepp, Science, 2017, 356, 496-497.

11 M.-C. Kim, E. Sim and K. Burke, Phys. Rev. Lett., 2013, 111, 73003.

12 R. G. Parr, in Horizons of Quantum Chemistry, Springer, 1980, pp. 5-15.

13 R. Ahlrichs, M. Bär, M. Häser, H. Horn and C. Kölmel, Chem. Phys. Lett., 1989, 162, $165-169$.

14 K. Eichkorn, O. Treutler, H. Öhm, M. Häser and R. Ahlrichs, Chem. Phys. Lett., 1995, 
240, 283-290.

15 F. Weigend and M. Häser, Theor. Chem. Acc., 1997, 97, 331-340.

16 T. H. Dunning, J. Chem. Phys., 1989, 90, 1007-1023.

17 F. Weigend and R. Ahlrichs, Phys. Chem. Chem. Phys., 2005, 7, 3297-3305.

18 K. P. Jensen, J. Phys. Chem. A, 2009, 113, 10133-10141.

19 K. P. Jensen, B. O. Roos and U. Ryde, J. Chem. Phys., 2007, 126, 14103.

20 D. Lee, F. Furche and K. Burke, J. Phys. Chem. Lett., 2010, 1, 2124-2129.

21 A. Wasserman, J. Nafziger, K. Jiang, M.-C. Kim, E. Sim and K. Burke, Annu. Rev. Phys. Chem., 2017, 68, 555-581.

22 K. P. Kepp, J. Phys. Chem. A, 2014, 118, 7104-7117.

23 A. Klamt and G. Schüürmann, J. Chem. Soc. Perkin Trans. 2, 1993, 799-805.

24 A. Schäfer, A. Klamt, D. Sattel, J. C. W. Lohrenz and F. Eckert, Phys. Chem. Chem. Phys., 2000, 2, 2187-2193.

25 S. Grimme, J. Antony, S. Ehrlich and H. Krieg, J. Chem. Phys., 2010, 132, 154104.

26 K. P. Jensen, Inorg. Chem., 2008, 47, 10357-65.

27 J. P. Perdew, A. Ruzsinszky, J. Tao, V. N. Staroverov, G. E. Scuseria and G. I. Csonka, J. Chem. Phys., 2005, 123, 62201.

28 A. D. Becke, J. Chem. Phys., 1993, 98, 5648-5652.

29 P. J. Stephens, F. J. Devlin, C. F. Chabalowski and M. J. Frisch, J. Phys. Chem., 1994, 98, $11623-11627$. 
30 C. Lee, W. Yang and R. G. Parr, Phys. Rev. B, 1988, 37, 785.

31 Y. Zhao and D. G. Truhlar, Theor. Chem. Acc., 2008, 120, 215-241.

32 J. P. Perdew, K. Burke and M. Ernzerhof, Phys. Rev. Lett., 1996, 77, 3865.

33 S. H. Vosko, L. Wilk and M. Nusair, Can. J. Phys., 1980, 58, 1200-1211.

34 T. Kato, Commun. Pure Appl. Math., 1957, 10, 151-177.

35 K. P. Jensen and U. Ryde, Coord. Chem. Rev., 2009, 253, 769-778.

36 K. Jensen and U. Ryde, J. Phys. Chem. A, 2003, 155, 7539-7545. 